\title{
The Relationship between Irritable Bowel Syndrome, Depression, Anxiety, and Stress among a Sample of Irritable Bowel Patients - Predictive Study
}

\author{
Osama Hasan Gaber \\ Associate Professor of Psychology at Faculty of Education \\ Dept. of Education and Psychology Najran University \\ P.O.Box 1988, Najran 61441, KSA \\ Tel: 00966561380347 E-mail: dr.osamagaber@yahoo.com
}

This research is funded by the Deanship of the Scientific Research at Najran University (NU/SHED/14/081)

Received: January 28, 2016 Accepted: February 13, 2016 Published: February 28, 2016 doi:10.5296/jsr.v7i1.9062 URL: http://dx.doi.org/10.5296/jsr.v7i1.9062

\begin{abstract}
This study aimed to examine the relationship of irritable bowel syndrome, anxiety, depression and stress among a sample of78 IBS patients (Rome III Diagnostic Criteria for Irritable Bowel Syndrome), anxiety symptoms scale ,Depression symptoms scale , and stress scale (prepared by the researcher) were used Pearson Correlation Coefficient showed that there are statistically significant relationship between IBS and Anxiety ,Depression and Stress $(\mathrm{P} \leq=0.01)$.The Regression and Prediction Coefficient (stepwise) was also used and showed that the depression and Stress of predicts to IBS.
\end{abstract}

Keywords: Irritable bowel syndrome- Depression- Anxiety- Stress 


\section{Introduction}

Irritable bowel syndrome (IBS) has been widely studied in the western world and pathphysiologic mechanisms have been available to explain the constellation of symptoms. IBS is a chronic disorder of unknown etiology clinically consisting of altered bowel habits, abdominal pain and the absence of any detectable organic pathologic process. Along with the putative pathophysiologic mechanisms of post-infectious inflammatory disorder, disordered intestinal motility and visceral hypersensitivity, there has been much discussion regarding the brain-gut interaction and the influence of behavioral or psychiatric conditions on these symptoms (Ladep et al 2006)

Irritable bowel syndrome (IBS) is a functional digestive disorder characterized by abdominal pain, bloating and altered bowel habits without any organic cause. Patients with IBS exhibit enhanced perception of visceral sensation to colonic distension which is associated with hypervigilance at the origin of visceral hyper sensitivity (VHS) .VHS is a clinical marker of IBS considered to play a major role in its pathophysiology. The exact cause of VHS is unknown but a number of mechanisms are evoked as represented by neuroplastic changes in primary afferent terminals (peripheral sensitization) due to peripheral inflammation or infection of the gut (i.e. post-infectious IBS) but also in the spinal cord (central sensitization) and in the brain (supraspinal pain modulation) or in descending pathways that modulate spinal nociceptive transmission In addition, stress is able to increase visceral sensitivity either at the central and/or peripheral level (Bonaz et al2012)

Several groups have determined that psychosocial stress alters GI motor function and sensation. In this way, psychosocial stressors likely exacerbate GI symptoms in patients who have functional GI disorders. Anxiety disorders, somatoform disorders, or a history of physical or sexual abuse can be identified in approximately $42 \%$ to $61 \%$ of patients who have IBS in referral practices. In particular, several studies suggested that the presence of somatization is particularly common and likely influences outcomes in patients who have IBS, The role of emotional stress as an etiologic factor in the development of IBS symptoms also may be inferred from the effects of psychologic therapies on IBS symptoms (Cash and Chey.2004)

Patients with IBS have been shown to have poor health-related quality of life (QOL) when compared with healthy individuals and often experience a significant financial burden-indeed, their economic impact factor increases from 1.1 to 6.0 when compared with non-IBS individuals . The characteristic pathophysiological features of IBS are dysmotility, visceral sensitivity, and psychological disturbances . The GI symptoms of IBS may aggravate depression and anxiety and be associated with psychosocial stress; indeed, patients with IBS symptoms improve with recovery from psychological disturbances. At least two studies have shown that patients with IBS report a high frequency of comorbid anxiety disorders such as panic disorder, agoraphobia, generalized anxiety disorder, and post-traumatic stress disorder (Saigo et al2014). Several clinical studies and reports from different researchers have shown that, among patients seeking medical attention for IBS, 70-90\% may have psychiatric co-morbidity, most commonly mood disorders, anxiety 
disorders and somatization disorder and this co- occurrence has added to controversy about the validity of the IBS diagnosis (Tosic et al .2010)

An early description of IBS more than 100 years ago made mention of associated psychological distress, A striking feature of this syndrome is the frequent finding of comorbid psychiatric illness. Studies suggest that $54 \%$ to $100 \%$ of patients with IBS have associated psychiatric illness (Thompson,1994). According to (Drossman et al1982), over one-half of individuals who met the criteria for a functional bowel disorder had never been Psychological stress is widely believed to play a major role in IBS. More than half of IBS patients and non patients (with symptoms compatible with IBS) report that psychologically stressful events.

exacerbate their bowel symptoms; $51 \%$ of IBS patients report that a stressful event preceded the onset of their disorder(Whitehead, Crowell.1991). (Walker et al 1990 )found that the onset of psychiatric symptoms occurred before the onset of gastrointestinal distress in the majority of these patients. The relative lack of psychiatric illness in a comparison group of patients with inflammatory bowel syndrome (19\%) suggests that the magnitude of psychiatric distress in IBS cannot be explained as a reaction to chronic gastrointestinal distress .

\section{Objective}

Considering the background above, this research aimed to:

a- examine the relationship between irritable bowel syndrome and depression

b- examine the relationship between irritable bowel syndrome and anxiety

c- examine the relationship between irritable bowel syndrome and stress

d- examine the predictive relationship for irritable bowel syndrome through anxiety, depression and stress.

\section{Literature Review}

\subsection{Irritable bowel syndrome}

The irritable bowel syndrome (IBS) is the most common disorder encountered by gastroenterologists. IBS is defined as "a functional bowel disorder in which abdominal pain is associated with defecation or a change in bowel habit with features of disordered defecation and distension"(Drossman 1999b)

Irritable bowel syndrome is a disorder characterized most commonly by cramping, abdominal pain, bloating, constipation, and diarrhea. IBS causes a great deal of discomfort and distress, but it does not per manently harm the intestines and does not lead to a serious disease, such as cancer. Most people can control their symptoms with diet, stress management, and prescribed medications. For some people, however, IBS can be disabling. They may be unable to work, attend social events, or even travel short distances. As many as 20 percent of the adult population, or one in five Americans, have symptoms of IBS, making it one of the most common disorders diagnosed by doctors. It occurs more often in women than in men, and it 
begins before the age of 35 in about 50 percent of people. be mucus in it, which is a fluid that moistens and protect passages in the digestive system. Some people with IBS experience diarrhea, which is frequent, loose, watery, stools. People with diarrhea frequently feel an urgent and uncontrollable need to have a bowel movement. Other people with IBS alternate between constipation and diarrhea. Sometimes people find that their symptoms subside for a few months and then return, while others report a constant worsening of symptoms over time. ( National of Institutes of Health2016)

The pathophysiology of IBS is still inadequately understood, but it is most likely due to complex interactions between the immune, hormonal and nervous systems. Diverse factors, including psychological stress, food intolerance or allergy, intestinal infection, injury (e.g., abdominal or pelvic surgery), intestinal immune disruption and/or inflammation, changes in the intestinal microbiota or bacterial overgrowth, and genetic transmission, abuse and early life learning, have been found to contribute to the development of IBS syndrome according to the research in the last decade.( Yan et al 2014)

The syndrome has been defined according to Rome III criteria. IBS affects up to $10-15 \%$ of the population with an estimated 1.7 billion dollars in annual direct cost. Generally patients suffer from the absence of a real diagnostic and from the consideration that they have a psychosomatic disease. Pain is perceived by patients as the most distressing symptom and constitutes their major reason for consulting a physician. Extra-intestinal manifestations are also frequently described by the patients (e.g. headache, low back pain, chronic fatigue, interstitial cystitis...) (Bonaz et al 2012). The pathophysiology of IBS is incompletely understood and thought to be multifactorial, Factors that may contribute to IBS include genetic, abnormal GI motility, visceral hypersensitivity, low-grade mucosal in flammation, impaired epithelial barrier function and alterations in intestinal flora; it is not known how these factors interact with each other.( Hayes et al. 2014)in (James.2015). There is evidence to suggest that there is a role for genetics in IBS, some studies have shown that concordance of IBS is twice as great in monozygotic compared with dizygotic twins, An association between IBS and psychological factors such as stress has also been described for many years(James.2015).Patients with IBS are suffering from a variety of GI complaints, as well as, associated symptoms like headache, dysuria, fibromyalgia, anxiety, depression, and chronic fatigue syndrome. Anxiety, depression and somatoform disorders are the most prevalent psychiatric conditions linked to IBS. It is reported that at least one of these disorders is present in approximately 40 to 60 percent of IBS patients visited by physicians in gastroenterology clinics. Results from some studies with population-based design have reported lower rates of psychiatric symptoms in non-consulters IBS patients (i.e., individuals who do not consult with any physician). While a number of IBS patients feel that their psychiatric disorders is the result of the intrusive nature of their IBS, others think that their psychiatric disorders are related to the development of their GI symptoms. However, approximately half of patients with a psychiatric disorder develop the condition before the onset of GI symptoms, and psychiatric symptoms start at the same time in most of the remaining fifty percent .( Farzaneh et al.2013).

The idea that emotions may influence the sensorimotor function of the gastrointestinal tract 
emerged at the beginning of the 19th century, and a lot of the evidence from research during that period is still valid. Nevertheless, the vivid part of the history of IBS began only three decades ago, when the concept of the biopsychosocial model of illness and disease was developed. This model integrates all possible accountable factors for the pathogenesis and clinical expression in IBS. The biopsychosocial approach allows for symptoms to be both determined and modified by psychological and social influences. The link between psychosocial factors and gastrointestinal function (motility, sensation, inflammation) is through the brain-gut axis. This implies a bidirectional connection system between the gastrointestinal tract and the brain, through neural, neuroimmune and neuroendocrine pathways(Belga et al 2012) . According to (Drossman 2006 ) in(Belga et al 2012)the Psychosocial factors influence every component of the biopsychosocial model . Early in life, genetics and environmental factors (e.g., family influences, abuse, major losses), may affect one's psychosocial development (psychological state, coping skills) and/or the development of gut dysfunction. Gut dysfunction and dysregulation of the braingut axis can lead to IBS. During life, psychosocial factors (stressful life events, psychological distress), may influence digestive function, symptom perception, illness behavior, and consequently health outcome, daily function and quality of life. Conversely, visceral pain can affect central pain perception, mood and behavior.

has been diagnosed most often by exclusion and has not yet been identified by any specific anatomical, physiological or biochemical marker. Various hypotheses about factors playing a part in the development of IBS include altered bowel motility, visceral hypersensitivity, neurotransmitter imbalance, infection psychosocial factors and dietary factors. It has been observed that only about $25 \%$ of persons with IBS seek help. Patients who seek medical help are more likely to have behavioral and psychiatric problems than those who never sought help for their bowel symptoms. About $60 \%$ of patients seen at referral centres have psychiatric symptoms of somatization, depression and anxiety. It is still not understood whether psychopathology is a cause or effect of it. But treating the coexisting psychopathology definitely improves the patient's symptoms.( Lanka, Bhogaraju.2013)

The fact that so many people with IBS have anxiety and depression has led many to speculate that IBS is primarily a psychological, not a physical, disorder. However, the condition is complex and most likely results from an interplay between psychological and biological factors. In fact, we don't really know whether anxiety and depression result from having IBS or whether they contribute to the development or maintenance of symptoms. In many patients, both possibilities may be true at the same time.( Elsenbruch.2014)

Through previous offer, we find that he needs to be more research shows that psychological factors that predict for irritable bowel syndrome, and this is the objective of the current study, they tested the predictive relationship for irritable bowel syndrome through anxiety, depression and stress. 


\subsection{Anxiety}

Anxiety disorders, as their name implies, are disorders associated with excessive feelings of anxiousness and nervousness. As with Major Depressive Disorder, Anxiety Disorders are simply a more extreme case of what all of us experience at various times. A person suffering from an anxiety disorder experiences anxiety in such a way that it significantly interferes with normal functioning. As you might suspect these cognitive feelings of anxiety and nervousness, neurologically, translate into an overly active sympathetic nervous system. In the case of Generalized Anxiety Disorder, these feelings of anxiousness and nervousness are constant, as is the sympathetic activation. Not only can such feelings be unpleasant, but they can also have a strong negative impact on our physical health. Thus, this type of anxiety disorder can be particularly debilitating. Panic disorder also includes anxious and nervous feelings, but these feelings come and go at unanticipated times. In fact, one of the defining components of Generalized Anxiety Disorder and Panic Disorder is that in neither case is the anxiety due directly to any specific external stimuli. The prototypical panic attack includes many physical components such as irregular heart rate, dizziness, faintness, shortness of breath, sweating and "clamminess". All of these are often associated with a general feeling of dissociation or unreality and even a feeling of impending death. Again, as you might suspect, a person suffering from such attacks, which com with little warning, can have significant problems in day to day functioning.(Hall.1998).

Recent work suggests that some patients may be biochemically more sensitive to the development of anxiety symptoms in the presence of particular diseases. patients with generalized anxiety disorders have higher plasma catecholamine levels than normal controls. These patients may down regulate catechol receptors as a result of these higher plasma concentrations and thus experience reduced receptor sensitivity in their adrenergic nervous system.(Hall et al .1997)

It is difficult to neatly compartmentalize organically induced from psychogenically induced anxiety symptoms in medically ill patients, as anxiety symptoms are ubiquitous in medical outpatient clinics and in patients admitted to hospital with severe disease. Estimates of significant anxiety in an outpatient clinic range from 10 to $20 \%$ of patients. (Wells et al1.988) found that more than $11 \%$ of persons with chronic medical conditions experienced a recent anxiety disorder. These findings were drawn from conclusions based on the review of a sample of 2554 patients with one of eight chronic medical conditions. These researchers found that patients with chronic medical conditions had a significantly higher adjusted lifetime prevalence of anxiety disorder than did those without. Their results were statistically significant for the presence of recent anxiety disorders at the $\mathrm{P}<0.005$ level. In trying to determine whether an underlying medical illness physiologically causes the patient's psychiatric symptoms as compared to the anxiety symptoms representing a reaction to the stress of the illness, Schuckit1983 applied some of the criteria noted above.17 He found that between 10 and $40 \%$ of medical patients with anxiety disorder had what he considered to be an organic etiology for their psychiatric symptoms.( Hall ,Ryan .1997). Also According to Harvard Medical School, there may be a connection between anxiety disorders and the development of irritable bowel syndrome (IBS) after a bowel infection. IBS can cause 
vomiting, diarrhea, or constipation.( Pietrangelo.2014)

About $10 \%$ to $20 \%$ of Americans suffer from the two most common functional digestive disorders - IBS and functional dyspepsia (upset stomach). In these disorders, the nerves regulating digestion appear to be hypersensitive to stimulation. Because these conditions don't produce lesions like ulcers or tumors, they aren't considered life-threatening. But their symptoms - abdominal pain, bloating, and diarrhea or constipation in IBS; and pain, nausea, and vomiting in functional dyspepsia - can be chronic and difficult to tolerate. There are no firm data on the prevalence of anxiety disorders in people with functional digestive disorders, but a 2007 New Zealand study of subjects with gastroenteritis (inflammation of the digestive tract) found an association between high anxiety levels and the development of IBS following a bowel infection.(Harvard Medical School.2008)

Although it is unlikely that psychiatric disorders are the single cause of IBS, they may be important cofactors in the development of the illness in susceptible people. A number of studies support this hypothesis, having found that the presence of upper GI symptoms in IBS patients increases the incidence and severity of psychopathology. Gwee at al1999 in( Farzaneh et al.2013) found that those who subsequently developed IBS had higher levels of anxiety, depression, somatization and neurotic personality traits than those who did not develop the illness.

\subsection{Depression}

Depression is a significant contributor to the global burden of disease and affects people in all communities across the world. Today, depression is estimated to affect 350 million people. The World Mental Health Survey conducted in 17 countries found that on average about 1 in 20 people reported having an episode of depression in the previous year. Depressive disorders often start at a young age; they reduce people's functioning and often are recurring. For these reasons, depression is the leading cause of disability worldwide in terms of total years lost due to disability. The demand for curbing depression and other mental health conditions is on the rise glob- ally. A recent World Health Assembly called on the World Health Organization and its member states to take action in this direction (Marcus et al 2012)

Depression is undoubtedly the most common mental health problem, especially when its milder form - dysphoria - is also included. In the United States, it is often called "The common cold of mental health." Most people occasionally are affected and in most cases it is self-limiting. But sometimes it is not self-limiting and requires psychological or psychiatric treatment and it is certainly more serious than a cold(Dowd.2004). The Depression According to( DSM-IV-TR2000) is During periods of depressed mood, at least two of the following additional symptoms are present; poor appetite or overeating, trouble sleeping or too much sleeping, low energy or fatigue, low self-esteem, poor concentration or difficulty making decisions, and feelings of hopelessness.

The original model of depression as developed by Aaron T. Beck saw it as characterized by a negative view of the self (e.g. "I am a loser"), the world (e.g. people will hurt me if I give them a chance"), and the future (e.g. things will never get better, only worse"). Beck was 
careful not to say that these dysfunctional thinking patterns necessarily caused depression (though they might in some circumstances) but that they were associated with depression. A complex mixture of biological variables, social factors, and psychological aspects likely causes depression. But there appears to be a cycle of cognition, affect or emotion, and behavior resulting in what has been called the downward spiral of depression. For example, the thought, "I am a hopeless loser" in response to a bad life event such as the loss of employment can result in feelings of sadness and apathy. Therefore the individual does not even look for other employment that in turn leads to further negative self-talk as well as further depressive feelings. But it is not even certain what causes cognitive change in cognitive behavior therapy .( (Dowd.2004).

Historically, three major theoretical models of depression have dominated throughout the 20th century: biological, intrapsychic and environmental approaches. Biological approaches posit that vulnerability to depression is a result of chemical imbalances. Specifically, biological models emphasis disequilibrium of neurotransmitters alluding to depressive symptoms being a brain-related dysfunction. While these reductionist models are still currently present within the medical model, in part because pharmacological treatment for depression can be successful, these models co-exist with diathesis-stress approaches . Intrapsychic models first gained popularity when Sigmund Freud founded the psychoanalytic approach to understanding psychopathology. Freudians assume that emotional disturbances and disorders originate due to unresolved internal conflicts from ones childhood. Modern versions of the intrapsychic wave followed with Beck's cognitive model and Blatt's model of depressive personality styles. These models changed the scope of the intrapsychic perspective and focused largely on how individuals perceive themselves and the world. An individual's perspectives were, therefore, viewed as a product of experience and learning . Modern versions of intrapsychic models currently exist and are continually tested and refined.(Alberta.2006). According to Biological Theories of Depression For decades theorists have pondered genetic predisposition to mood disorders.Research on mood disorders having a heritable component has offered some support for this predisposition. Success rates of pharmacological treatments of depression, such as selective serotonin reuptake inhibitors, suggest the importance of biological factors (Davison et al.2005)

The Results of Some Studies indicates to Depression can affect the appetite. Some people cope by overeating or binging. This can lead to weight and obesity-related illnesses like type 2 diabetes. Others lose their appetite or fail to eat nutritious food. Eating problems can lead to stomachaches, cramps, constipation, or malnutrition. Symptoms may not improve with medication.(Pietrangelo.2014) Depression symptoms often include certain digestive problems research has shown that the makeup of bacteria in your gut can influence your emotional health. However, not all tummy troubles are due to a mental health condition.

Depending on your symptoms, you could have an inflammatory bowel disease (IBD) or irritable bowel syndrome (IBS), two types of gastrointestinal (GI) conditions, according to the Crohn's \& Colitis Foundation of America (CCFA. The Patients with depressive symptoms typically have reported a lower quality of life and may be at an increased risk for relapses of IBD symptoms. Depression can directly impact the healing process for IBD because it affects 
the immune system, which can prompt an inflammatory response. "Studies have found that when patients [with IBD] received mental health care, they spent fewer days in the hospital and took fewer sick days off from work(Sohrabi.2016)

\subsection{Stress}

Stress as a specific medical term was first defined by the endocrinologist Hans Selye in 1936as the physiological adaptive responses to perceived (psychological) or real (physical) threats ("stressors") to an organism. An acute (sudden or short-term) stressor can evoke a "fight or flight" response that prepares to defend the stability of the internal environment in order to ensure the survival of the organism. When the stress passes, a negative feedback is triggered to terminate the stress response and bring the body back to a state of homeostasis or eustasis. However, if the stressor becomes chronic and/or exceeds the organism's ability to maintain the stress response, it becomes harmful because basal homeostasis cannot be reached. For most humans in modern societies, psychological stress is more frequent than physical stress and it may be induced by various social and emotional triggers, some of which can be unique for an individual.( Yan et al 2014). Stress can affect bowel function in anyone, but the effect is greater in people with IBS due to changes in the brain-gut axis that affect the brain's ability to reduce the body's reaction to stress. It has also been shown that many people with IBS have a history of early trauma, including physical or sexual abuse. This can cause a person to be more sensitive and vulnerable to body sensations.( Christine.2016a)

It has been a popular notion that the manifestations of IBS include emotional and perceptual components, which are suggestive of the brain-gut interaction. Several studies have shown an alteration in bowel function during sleep and specific sleep disturbances in patients with IBS, suggesting that altered CNS function may be playing an important role in the pathogenesis of IBS. Using PET (positron-emission tomography) scanning, it appears that the activated portions of the brain cortex by the stimulation of sigmoid distention in patients with IBS .The enteric nervous system contains almost as many neurotransmitters as the brain itself. These transmitters form important relation- ships between gut function and stress. (Sasaki.2003) .

Stress is known to decrease gastric emptying, lengthen small bowel motility and increase colonic motility. The effects of stress on gut function are mediated by the ANS represented by the sympathetic, vagal and pelvic parasympathetic innervations of the enteric nervous system . At the central level, stress inhibits the parasympathetic nervous system and activates the sympathetic nervous system through the effect of PVN projections on the DMNV and intermediolateral column cells of the spinal cord.( Bonaz et al 2012)

Acute stress and emotional arousal may induce the change of the colonic motilities. Colonic motilities show exaggerated patterns when IBS patients are under the stress. There are no specific findings of the colonic motility pattern seen in IBS, but several principal motility abnormalities are seen in it. IBS patients report pains to be less intense when a balloon is inflated in the colonic lumen. It is assumed that visceral hyperalgesia is seen in IBS patients due to the decrease of the perceptual sensitiv- ity threshold induced by intraluminal balloon distention. (Whitehead et al) examined by meta- analysis whether visceral heperalgesia is a diagnostic biological marker of IBS, and psycho- logical factors have an influence on 
perceptual sensitivity in IBS patients or not. Many of these findings cannot be explained on the basis of biological differences between patients with IBS and controls. It may be confirmed that psychosocial stressors have an influence on the pain threshold in patients with IBS.(see Sakaki.2003)

Stress also induces an activation of the sacral parasympathetic nucleus through the projections of the Barrington nucleus through CRF activation thus stimulating recto-colonic motility (Bonaz and Tache 2007). Numerous data have established the involvement of peripheral CRF signalling in the modulation of secretory function under stress conditions via activation of both CRF1 and CRF2 receptors, activation of cholinergic enteric neurons, mast cells and possibly serotonergic pathways (Mulak et al 2012)

The impact of stress on the stomach goes far beyond indigestion, however. In recent years, doctors have uncovered a remarkably complex connection between the brain and the digestive system. The entire system is extremely sensitive to our moods. In fact, experts now see stress as a major player in a wide range of digestive problems, including irritable bowel syndrome, indigestion, and heartburn .People with digestive problems often scoff at the idea that stress could be at the root of their problems.( Woolston.2016)

\section{Research Issue}

This study aimed to examine the relationship between irritable bowel syndrome and depression, examine the relationship between irritable bowel syndrome and anxiety ,examine the relationship between irritable bowel syndrome and stress and examine the predictive relationship for irritable bowel syndrome through anxiety, depression and stress .The research inquiries are:

a. What is the relationship between depression and the irritable bowel syndrome of IBS patients?

b. What is the relationship between anxiety and the irritable bowel syndrome of IBS patients?

c. What is the relationship between stress and the irritable bowel syndrome of IBS patients?

d. Can irritable bowel syndrome be predicted by depression, anxiety or stress?

\section{Methodology \& Procedures}

\section{5-1 Methodology}

Descriptive method is used. It enables the researcher to explore the relationship of irritable bowel syndrome and anxiety, depression, stress and the predicted relationship irritable bowel syndrome and those variables.

\section{5-2 Study population and sample}

The study tools were applied on 78 irritable bowel syndrome patients . 


\section{5-3 Study tools}

The following tools were used:

- Anxiety symptoms Scale, prepared by the researcher.

- Depression symptoms Scale, prepared by the researcher.

- Psychological stress scale, prepared by the researcher.

- Rome III Diagnostic Criteria for Irritable Bowel Syndrome.

\section{Results \& Discussion}

6.1. The first inquiry: What is the relationship between Irritable Bowel Syndrome and the Depression for IBS patients

Table 1: Correlation coefficient and statistical significance between motivation Irritable Bowel Syndrome and the Anxiety for The study sample.

\begin{tabular}{|l|l|l|}
\hline Variables & Depression & Significance \\
\hline Irritable Bowel Syndrome & 0.379 & 0.01 \\
\hline
\end{tabular}

There is a positive statistical significance relation between Irritable Bowel Syndrome and depression at 0.01. This result shows that more depression, more Irritable Bowel Syndrome .According (Schmid et al2014) High rates of depression amongst patients with irritable bowel syndrome (IBS) have led many researchers to believe there could be a causal relationship between psychological factors and IBS symptoms. Now, scientists in Germany have found clear evidence that patients with IBS process pain signals from the gut abnormally, and that disturbed brain responses to pain are particularly pronounced in patients with more depression symptoms. may also be symptoms of depression. See a doctor for a medical evaluation to determine the cause of your symptoms. Digestive problems may also be symptoms of depression. See a doctor for a medical evaluation to determine the cause of your symptoms .When people are depressed, they sometimes develop digestive problems, including diminished appetite, nausea, diarrhea, and constipation. Worry, anxiety, and stress can lead to intestinal difficulties. Also, people experiencing a depressive episode may eat not enough or too much, and they may get little exercise. All this can affect the stomach and intestinal tract.( Goldberg.2016) Often have physical symptoms of depression, this is kind of the symptoms of the most common and most insidious symptoms, speed fatigue, poor energy, back pain and body, without that there will be reasons clear membership justify It somatic complaints of depression, sleep disorders, headaches, stomach pains and Digestive disorders .(Ibrahim.1990)

The evidence of relationship depression with irritable bowel disease research studies have shown that they can also be effective as analgesics (drugs that reduce pain). Antidepressants 
are, therefore, used to treat such chronic painful conditions as migraine headaches, diabetic neuropathy and fibromyalgia. Similarly, antidepressants are effective in treating symptoms of IBS and other functional GI disorders. Patients who have taken antidepressants for their IBS symptoms have reported significant improvement in their abdominal pain and reduction in other IBS symptoms, such as diarrhea, constipation, bloating, nausea or urgency. The brain is always monitoring and processing all that goes on in the body. As already noted, antidepressants are known to work at the level of the brain and spinal cord to block pain messages between the GI tract and the brain, thereby reducing visceral hypersensitivity. In effect, there is recovery of more normal brain-gut function, possibly by helping the brain send down signals to block incoming pain impulses, a natural response. Much like treating diabetes with the insulin that is missing, antidepressants may help recover the brain's ability to respond to pain signals properly. Certain antidepressants can also help regulate abnormal bowel functions like diarrhea and, constipation, as well as other IBS symptoms. The tricyclic antidepressants (TCA's) help with diarrhea and the serotonin reuptake inhibitors (SSRI's) help treat constipation. Furthermore, these medicines can help with other problems such as anxiety and depression, which are often associated with chronic painful disorders.(Christine et al 2016b)

6.2. The second inquiry: What is the relationship between Irritable Bowel Syndrome and the Anxiety for IBS patients

Table 2: Correlation coefficient and statistical significance between Irritable Bowel Syndrome and the Anxiety.

\begin{tabular}{|l|l|l|}
\hline Variables & Anxiety & Significance \\
\hline Irritable Bowel Syndrome & 0.337 & 0.01 \\
\hline
\end{tabular}

There is a positive statistical significance relation between Irritable Bowel Syndrome and anxiety at 0.01 . This result shows that more anxiety, more Irritable Bowel Syndrome. According Gournay The causation of irritable bowel syndrome is something that remains the source of debate and controversy. However, it seems clear from the physiological point of view, that some people have particularly sensitive intestinal tracts and muscle activity is increased. There is also some evidence that some people are particularly sensitive to certain foodstuffs. Without doubt, anxiety is a factor that can increase bowel activity and, thus, it is likely in most cases that there is a mixture of both physiological and psychological components to causation. However, it should also be said that in some people the anxiety component of causation may be minor, while in other cases, anxiety is a very substantial factor. Thus, psychological factors, such as anxiety, may be important in increasing or even causing irritable bowel syndrome and the irritable bowel syndrome itself leads to secondary anxiety because of the obvious problems associated with having such symptoms. It must be said at this point that there is some evidence that other psychological approaches have been shown to be helpful in the treatment of irritable bowel syndrome and, for some patients, it is clear that some forms of psychotherapy seems to have an effect. Having said this, the 
behavioural management of the bowel problem itself is important and a purely psychological approach to the problem is unlikely to be of benefit to the vast majority of patients.(Gournay.2016). Much less is known about the impact of anxiety disorders on function and outcome in persons with chronic medical illness. There is convincing evidence that anxiety is associated with high rates of medically unexplained symptoms and increased utilization of healthcare resources(Katon and Kroenke.2007)

In recognition of the need to better understand and illustrate the effect of anxiety disorders on persons with chronic medical illnesses and with the hope of developing treatment strategies, the Anxiety Disorders Association of America (ADDA) convened a multidisciplinary conference on January 30-31, 2006, to review current data on the relationship between anxiety disorders and specific medical illnesses. Presenters and discussants included clinicians and researchers in psychiatry, psychology, primary care, health- care systems, epidemiology, public health, healthcare policyand advocacy. The proceedings of the conference are summarized in this paper, which reviews anxiety disorders in the context of functional gastrointestinal disorders, asthma, heart disease, cancer and chronic pain. These selective reviews mainly focused on anxiety disorders per se and did not investigate numerous studies focused on"stress". In addition, specific recommendations are made for furthering the basic science and clinical research agenda to better understand the impact of anxiety disorders on medical illness and improve clinical outcomes and patient care(Byrne et al 2008).Also here is a strong association between IBS and psychiatric diagnoses, particularly anxiety disorders. Rates of psychiatric diagnoses range from 54\% to $94 \%$ in treatmentseeking patients with IBS . Rates of anxiety and mood disorders in patients with IBS are significantly higher than in patients with inflammatory bowel disease . When psychiatric disorders co-exist with IBS, gastrointestinal symptoms are typically more severe and disabling. In a survey of university students, IBS was associated with high rates of generalized anxiety disorder (GAD) and higher levels of neuroticism, visceral anxiety, anxiety sensitivity and worry than in those without.( Drossman et al 2002)

\subsection{The Third inquiry: What is the relationship between Irritable Bowel Syndrome and stress for IBS patients}

Table 3: Correlation coefficient and statistical significance between Irritable Bowel Syndrome and the stress.

\begin{tabular}{|l|l|l|}
\hline Variables & Stress & Significance \\
\hline Irritable Bowel Syndrome & 0.360 & 0.01 \\
\hline
\end{tabular}

There is a positive statistical significance relation between Irritable Bowel Syndrome and stress at 0.01. This result shows that more stress, more Irritable Bowel Syndrome, Stress as a specific medical term was first defined by the endocrinologist Hans Selye in 1936 as the physiological adaptive responses to perceived (psychological) or real (physical) threats 
("stressors") to an organism. An acute (sudden or short-term) stressor can evoke a "fight or flight" response that prepares to defend the stability of the internal environment in order to ensure the survival of the organism. When the stress passes, a negative feedback is triggered to terminate the stress response and bring the body back to a state of homeostasis or eustasis. However, if the stressor becomes chronic and/ or exceeds the organism's ability to maintain the stress response, it becomes harmful because basal homeostasis cannot be reached[9]. For most humans in modern societies, psychological stress is more frequent than physical stress and it may be induced by various social and emotional triggers, some of which can be unique for an individual. (Mulak et al 2012)

According to( Tache 2015) a person's stress response involves a network of brain regions that interact as they receive information from inside or outside the body. When stress actives this network, it triggers two main pathways. One is called the pituitary-adrenal axis, which acts to increase circulating hormones (glucocorticoids - particularly cortisol) involved with regulating the body's response to stress. The other is the autonomic nervous system, which regulates involuntary bodily functions such as our blood pressure, heart rate, and bowel function. Both of these pathways directly or indirectly affect gut function through the unique system of nerves within the bowel wall (enteric nervous system). These pathways, along with the brain and enteric nervous system, are collectively referred to as the "brain-gut axis.

In the stress-activated pathways, the corticotrophin releasing factor (CRF) signaling system is a key element in the biochemical mechanism by which the brain trans- lates a stimulus into an integrated physical response. This system is composed of the 41 amino acid peptide, three related peptides, namely urocortin 1, urocortin 2 and urocortin 3, as well as the CRF receptors CRF1 and CRF2 and their variants[9]. When the body experiences stress, the CRF signaling system plays a primary neuroendocrine role in stimulating the HPA axis, acting as a neurotransmitter/neuromodulator to coordinate the immune and visceral efferent limbs, and activating the locus coeruleus and its noradrenergic projections. The CRF system can also modulate the forebrain, hindbrain and spinal sites for regulating the autonomic nervous system activity, leading to the stimulation of the sym- pathetic nervous system, release of catecholamines and induction of sacral parasympathetic activity. In addition, stress affects directly or indirectly the composition and the growth of microbiota, which helps to maintain bidirectional communication between the components of the brain and the gut axis. The impact of stress on the brain-gut axis has been reviewed by Grenham et al and O'Malley et al(see Yan et al 2014). Also The stress response causes a number of detrimental events in the gut, including Decreased nutrient absorption, decreased oxygenation to your gut ,as much as four times less blood flow to your digestive system, which leads to decreased metabolism and decreased enzymatic output in your gut - as much as 20,000-fold but that's not all . According as written in the featured Journal of Physiology and Pharmacology study:"Stress, which is defined as an acute threat to homeostasis, shows both short- and long-term effects on the functions of the gastrointestinal tract. The major effects of stress on gut physiology include alterations in gastrointestinal motility, increase in visceral perception ,changes in gastrointestinal secretion, negative effects on regenerative capacity of gastrointestinal mucosa and mucosal blood flowand negative effects on intestinal microflora (Mercola.2015) 
6.4. The fourth inquiry: Can Irritable Bowel Syndrome be predicted by depression .anxiety or stress

Regression coefficient and gradual prediction (stepwise) were used. There is two modes predicts the Irritable Bowel Syndrome by(1) Depression and (2) depression with stress

6-4-1 The model number one predicts the Irritable Bowel Syndrome by depression

Table 5: Model of regression coefficient and gradual prediction of Irritable Bowel Syndrome by depression

\begin{tabular}{|c|c|c|c|c|c|}
\hline \multirow{2}{*}{ Model } & \multicolumn{2}{|c|}{ Unstandardized Coefficients } & \multirow{2}{*}{$\begin{array}{l}\text { Standardized Coefficients } \\
\text { Beta }\end{array}$} & \multirow{2}{*}{$\mathrm{T}$} & \multirow{2}{*}{ Sig } \\
\hline & B & Std.Error & & & \\
\hline (Constant) & 25.782 & 8.507 & & 3.031 & .000 \\
\hline Depression & .581 & 0.172 & .379 & 3.379 & .000 \\
\hline
\end{tabular}

Multiple regression equation of the model:

Dependent Variables (Irritable Bowel Syndrome)= Constant+ B for (Depression) $\mathrm{x}$ value Depression Example: if it is assumed that one Irritable Bowel Syndrome patient received a score of 50 on the Depression scale, her/his score of Irritable Bowel Syndrome will $=25.782$ $+(0.581 \times 50)$

$=25.782+29.05$

$=54.832$

These result mean that Irritable Bowel Syndrome predict by depression, Such an these result consistent with the results of a study of (Benett et al1998) Which found Chronic life stress threat was a powerful predictor of subsequent symptom intensity, explaining $97 \%$ of the variance on this measure over 16 months. No patient exposed to even one chronic highly threatening stressor improved clinically (by 50\%) over the 16 months; all patients who improved did so in the absence of such a stressor.

Stress is a ubiquitous condition that affects all people. Stress can be mental or physical, although in the context of this article the focus will be mental stress. Mental stress involves challenge, threat or worry about future adverse events. Such stress activates the brain's stress response systems, which in turn affect the body. Many of the body's major systems are altered by stress (cardiovascular, muscular, urinary, gastrointestinal, sweat glands, etc) often with adverse consequences.

Two of the primary brain regions involved in stress reactivity are the hypothalamus and the 
locus ceruleus. Activation of the hypothalamus by stress is likely to be mediated in part by the limbic brain (particularly the amygdala and hippocampus) and partly by the locus ceruleus in the brainstem. The locus ceruleus and the hypothalamus actually stimulate each other, creating the potential for a vicious cycle, where a stress reaction in one region stimulates the other, which in turn stimulates the first to react even more. The limbic system is a group of connected and related brain regions that mediate emotions and flight or fight attitudes. The limbic or "emotional brain" is more primitive by evolutionary standards, and is not necessarily under control by the higher intellectual cortex. This system receives sensory and higher cortical inputs, calls upon memories and determines the threat level imposed by a stimulus. The amygdala for instance is a limbic structure in the base of the brain that is important in anger and rage. In cats, electrical stimulation of the amygdala causes hissing, back arching and the hair to stand on end, typical of anger and defense postures in cats. In animals that have damage to the amygdala a placid state results in which anger cannot be induced. Inputs to the amygdala are thought to originate from the hippocampus, the cingulate cortex and other parts of the limbic system. The locus ceruleus is located in the pontine portion of the brainstem. The locus ceruleus is the source of most of the stimulant neurotransmitter norepinephrine in the nervous system. Cells here project to other brain areas, releasing norepinephrine to activate other systems and increase arousal and alertness. Release of norepinephrine increases heart rate, blood pressure and primes the muscles and nervous system for fight or flight. This reaction is not helpful in routine stress of daily activities. If the stress reaction is excessive or the perceived threat too frequent, tachycardia (racing heart), hypertension, muscle tension, bowel spasms, and dyspepsia can result.(Mertz.2016)

6-4-2 The model number two predicts the Irritable Bowel Syndrome by depression with stress

Table 6: Model of regression coefficient and gradual prediction of Irritable Bowel Syndrome by depression with stress

\begin{tabular}{|c|c|c|c|c|c|}
\hline \multirow{2}{*}{ Mode2 } & \multicolumn{2}{|c|}{ Unstandardized Coefficients } & \multirow{2}{*}{$\begin{array}{l}\text { Standardized Coefficients } \\
\text { Beta }\end{array}$} & \multirow{2}{*}{$\mathrm{T}$} & \multirow{2}{*}{ Sig } \\
\hline & B & Std.Error & & & \\
\hline (Constant) & 11.192 & 9.485 & & 1.180 & .000 \\
\hline \multirow{2}{*}{$\begin{array}{l}\text { Depression } \\
\text { stress }\end{array}$} & 0.515 & 0.165 & 0.337 & 3.132 & .000 \\
\hline & .397 & 0.136 & 0.314 & 2.927 & .000 \\
\hline
\end{tabular}

Multiple regression equation of the model:

Dependent Variables (Irritable Bowel Syndrome) $=$ Constant+ B for (Depression) $\mathrm{x}$ value Depression $+\mathrm{B}$ for stress $\mathrm{x}$ value of stress 
Example: if it is assumed that one Irritable Bowel Syndrome patient received a score of 60 on the Depression scale and received a score of 54, her/his score of Irritable Bowel Syndrome will $=11.192$

$=11.192+0.515 \times 60+0.397 \times 54$

$=11.192+30.9+21.438$

$=63.53$

This result indicates that stress and depression predict IBS, Stress and depression were independent risk factors for FD and IBS in our large-scale population-based study, and this finding was consistent with that in several previous studies. This association is probably derived from mutual and reciprocal interactions between the brain and the gut. Corticotropin releasing hormone $(\mathrm{CRH})$, a major mediator of the stress response in the brain-gut axis, can increase intestinal permeability and lead to FD and IBS. In addition, serotonin and the serotonin transporters, which assist the modulation of feelings and behavior such as anxiety and depression, can be associated with brain-gut function in functional GI disorders.(Pyo et al.2015)

Negative affective and emotional states, such as anger, have been shown to be related to a decrease of antral motor activity in IBS patients, whereas in controls the same activity increased in anger-provoking situations. Intestinal motility patterns may suffer emotional stimulation and, particularly, an exaggerated gastrocolic reflex, altered gastric emptying, increased small intestinal transit and small bowel contractions following stressful events have been documented in IBS patients8. The proposed rationale for this vulnerability is that IBS patients may have a lower threshold for coping with stressful events and negative emotions, however, regarding defensiveness(Dibajnia et al 2013)

\section{References}

1- Alberta L (2006) Anxiety ,Depression and Hopelessness in Adolescents : A structural Equation Model, In Partial Fulfillment of the Requirements for the Degree Master of Education, faculty of Education, University of Lethbridge , Retrieved on 17/1/2016 from : http://www.uleth.ca/education/sites/education/files/ShaylynCunningham.pdf

2- Bennett J , Tennant C , Piesse C , Badcock A, Kellow J(1998) Level of chronic life stress predicts clinical outcome in irritable bowel syndrome, ritrived on 22/11/2046 from : http://gut.bmj.com/content/43/2/256

3- Blaga T, Băban A ,Dumitrascu D(Psychosocial determinants of irritable bowel syndrome, World J Gastroenterol; 18(7): 616-626

4- Bonaz B , Tache, Y (2007). " Corticotropin -releasing factor receptors and stress-related alterations of gut motor function." The Journal of clinical investigation 117(1): 33-40.

5- Bonaz,B \& Pellissier,S Sinniger,V Clarençon,D Peinnequin,A and Canini,F (2012) The Irritable Bowel Syndrome:How Stress Can Affect the Amygdala Activity and the Brain-Gut Axis, licensee In Tech .Ritrived on 11/1/2016 from : 
http://www.intechopen.com/download/pdf/41592

6- Byrne, M ,Karina W, Davidson, W, Ronald C, Gordon J, Asmundson D(2008) Anxiety disorders and comorbid medical illness, General Hospital Psychiatry 30208 225 , Ritrived on 15/1/2016 from

7- Christine B, Dalton, PA Douglas A, Drossman, M (2016a) Irritable Bowel Syndrome ,Center of functional GI \& Motility Disorder ,Retrieved on 4/1/2016 from : https://www.med.unc.edu/ibs/files/educational-gi-handouts/IBS.pdf

8- Christine B, Dalton, PA Douglas A, Drossman, $\mathrm{M}(2016 \mathrm{~b})(\mathrm{The}$ Use of Antidepressants in the Treatment of Irritable Bowel Syndrome and Other Functional GI Disorders, Center of functional GI \& Motility Disorder,Ririved on 28/1/2016 from : www.med.unc.edu/ibs/files/educational-gi-handouts/IBS and Antidepressants.pdf

9- Davison, G, Neale, J.M., Blankstein, K, Flett G (2005). Abnormal Psychology (2nd Eds). Mississauga, ON: John Wiley \& Sons Canada, Ltd.

10- Dibajnia P , Moghadasin,M, Madahi M and Keikhayfarzaneh M (2013 ) Depression, Anxiety, Stress, Anger in IBS Patients,Journal of Pure and applied microbiology, Vol.7(1) : 175-180

11-Dickhaus B, Mayer EA, Firooz N, (2003) Irritable bowel syndrome patients show enhanced modulation of visceral perception by auditory stress. Am J Gastroenterol :98:135-43.

12-Dowd T (2004) Depression: Theory, assessment, and new directions in practice, International Journal of Clinical and Health Psychology. Volume4 ,N2 :413-423

13- Drossman DA, Camilleri M, Mayer EA, Whitehead WE.(2002) technical review on irritable bowel syndrome. Gastroenterology;123:2108-31.

14- Drossman DA, Sandler RS, McKee DC, Lovitz HA. Bowel patterns among subjects not seeking health care: use of a questionnaire to identify a population with bowel dysfunction. Gastroenterology 1982;83:529-34.

15- Drossman DA. The functional gastrointestinal disorders and the Rome III process. In: Drossman DA, Corazziari E, Delvaux M, Spiller R, Talley NJ, Thompson WG, Whitehead WEeds. Rome III: the functional gastrointestinal disorders. 3rd ed. McLean, VA: Degnon Associates Inc, 2006: 1-30

16- Drossman, D.A. (1999). "The Rome criteria process: diagnosis and legitimization of irritable bowel syndrome." Am J Gastroenterol 94(10): 2803-2807.

17-DSM-IV-TR (2000) Diagnostic and Statistical Manual of Mental Disorders ,Fourth Edition, American Psychiatric Association, Washington ,DC

18- Dunphy RC, Verne GN. Drug treatment options for irritable bowel syndrome: managing for success. Drugs Aging 2001; 18: 201-211 
19-Elsenbruch S (2014) The Relationship between depression and abnormal brain response to visceral pain in patients with IBS, Ritrived on 1/1/2016 from : https://www.ueg.eu/press/releases/ueg-press-release/article/ueg-week-press-releasenew-st udy-finds-link-between-depression-and-abnormal-brain-response-to-viscer/

20- Farzaneh,N Ghobakhlou, M Moghimi,B Naderi ,N , Fadai ,F(2013) Anxiety and Depression in a Sample of Iranian Patients With Irritable Bowel Syndrome, Iran J Psychiatry Behav Sci, Volume 7, Number 1.

21-Fukudo ,S (2015) Evidence-based clinical practice guidelines for irritable bowel syndrome, J Gastroenterol 2015;50:11-30

22- Goldberg J (2015) WebMD's Symptom Finder: Physical Symptoms of Depression Stomach, Ritrived on 21/11/2015 from

http://www.webmd.com/depression/symptom-stomach

23-Gournay K (2016) Irritable Bowel Syndrome, Retrieved on 1/1/2016 from : http://www.nopanic.org.uk/ibs/

24-Hall R (1998) Anxiety Disorder ,Ritrived on 11/1/2016 from : http://web.mst.edu/ rhall/neuroscience/08_stress_anxiety/anxiety.pdf

25- Hall R , Ryan H (1997) Anxiety and Endocrine diseases Ritrived on 11/1/2016 from: http://www.drrichardhall.com/anxiety.htm

26-Harvard Medical School( 2008) Anxiety and physical illness , Harvard Health Publications ,Ritrived on 28/12/2015 from

http://www.health.harvard.edu/staying-healthy/anxiety_and_physical_illness

27-Hayes P, Fraher M, Quigley E, Irritable bowel syndrome(2014): the role of food in pathogenesis and management, Gastroenterology and Hematology;10(3):164- 174.

28-Horwitz BJ,(2011) Fisher RS. The irritable bowel syndrome. N Engl J Med; 344: 1846-1850

29- Jackson JL, O’Malley PG, Tomkins G, et al. Treatment of functional bowel gastrointestinal disorders with antidepressant medications: a meta-analysis. Am J Med 2000; 108:65-72.

30- James.S (2015) Irritable bowel syndrome, National Medicines Information Center ,Volume 21,Number 4,Ritrived on 5/1/2016 from: http://www.stjames.ie/GPsHealthcareProfessionals/Newsletters/NMICBulletins/NMICBu lletins2007/Travel\%20Medicine\%20(1)\%20Vol.13\%20No.1\%202007.pdf

31- Katon W, Lin EH, Kroenke K. The association of depression and anxiety with medical symptom burden in patients with chronic medical illness. Gen Hosp Psychiatry 2007;29:147-55.

32-Lanka U , Bhogaraju A(2013) A controlled study of psychopathology, life events, personality and sociodemographic factors in irritable bowel syndrome, AP J 
Psychological Medicine Vol. 14 (2) :124-132

33- Marcus M, Yasamy T, Ommeren M Chisholm D, Saxena S (2012 ) Depression - A Global Public Health Concern, Retrieved on 19/1/2016 from : http://www.who.int/mental_health/management/depression/who_paper_depression_wfmh _2012.pdf

34- Mercola (2015) How Stress Wreaks Havoc on Your Gut -- And What to Do About It,ritrivede on $28 / 12 / 215$ from http://articles.mercola.com/sites/articles/archive/2012/04/09/chronic-stress-gut-effects.asp $\mathrm{x}$

35- Mertz. H (2016) Stress and the Gut, Center of functional GI \& Motility Disorder, retrieved on 5/1/2016 from: https://www.med.unc.edu/ibs/files/educational-gi-handouts/Stress and the Gut.pdf

36- Mulak A, Larauche, M., Tache Y (2012). "Stress and visceral pain: from animal models to clinical therapies." Experimental neurology 233(1): 49-67.

37- National of Institutes of Health(2016) Irritable Bowel Syndrome, Department of Health and Human Services ,USA, Retrieved on 1/1/2046 from : http://www.niddk.nih.gov/health-information/health-topics/digestive-diseases/irritable-bo wel-syndrome/Pages/overview.aspx

38- Pietrangelo A (2014) The Effects of Depression on the Body ,Ritrived on 7/1/2016 from: http://www.healthline.com/health/depression/effects-on-body

39- Pyo S , Sung K , Hwan K, Young S , Seok H, Shim C (2015) The Effect of Emotional Stress and Depression on the Prevalence of Digestive Diseases, J Neurogastroenterol Motil21(2): 273-282.

40-Saigo, T Tayama,J Hamaguchi,T Nakaya,N Tomiie,T Bernick,P Kanazawa,M Labus,J D Naliboff,B Shirab,S and Fukud,S(2014) Gastrointestinal specific anxiety in irritable bowel syndrome: validation of the Japanese version of the visceral sensitivity index for university students,Journal of BioPsychoSocial Medicine, 8:10.

41-Sasaki D (.2003) Irritable Bowel Syndrome and Stress, Journal of the Japan Medical Association (Vol. 46, No. 2:66-69

42-Schmid, J. Langhorst, F. Gass, N. Theysohn, S. Benson, H. Engler, E. R. Gizewski, M. Forsting, S. Elsenbruch. Placebo analgesia in patients with functional and organic abdominal pain: a fMRI study in IBS, UC and healthy volunteers. Gut, 2014; DOI: 10.1136/gutjnl-2013-306648

43-Sohrabi F (2016) When Depression is a Symptom of Digestive Disease, Ritrived on $1 / 1 / 2016$ from http://www.everydayhealth.com/news/when-depression-symptom-digestive-disease/

44- Tache Y(2015) Stress and Irritable Bowel Syndrome: Unraveling the Code,ritrived on 
http://www.giresearch.org/site/iffgd-research-awards/2005/stress-and-ibs

45- Thompson WG. Irritable bowel syndrome: strategy for the family physician. Can Fam Physician 1994;40:307-15.

46- Tosic,S Golubovic,M Miljkovic,S Nagorni,A Lazarevic,D \& Nikolic,G (2010) Irritable Bowel Syndrome, Anxiety ,Depression and Personality Characteristics, Jourmnal of Psychiatria Danubina, 2010; Vol. 22, No. 3, pp 418-424

47- Walker EA, Roy-Byrne PP, Katon WJ, Li L, Amos D, Jiranek G. Psychiatric illness and the irritable bowel syndrome: a comparison with irritable bowel disease. Am J Psychiatry 1990;12:1656-61.

48- Whitehead WE, Crowell MD. Psychologic considerations in the irritable bowel syndrome. Gastroenterol Clin North Am 1991;20:249-67.

49- Woolston C (2016) Stress and the Digestive System, Ritrived on 26/1/2016 from : http://consumer.healthday.com/encyclopedia/digestive-health-14/digestion-health-news-2 00/stress-and-the-digestive-system-645906.html

50- Yan H , Wah C , Dong C , Zhao T(2014) Impact of psychological stress on irritable bowel syndrome, World J Gastroenterol ,Volume 20 ,Issue 39 : 1426-1431

\section{Copyright Disclaimer}

Copyright for this article is retained by the author(s), with first publication rights granted to the journal.

This is an open-access article distributed under the terms and conditions of the Creative Commons Attribution license (http://creativecommons.org/licenses/by/3.0/). 